\title{
Microgrid Islanding Detection Method based on Frequency - Reactive Power Feedback
}

\author{
Shibo Wang, Qi Wei and Siwen Cai \\ Electrical \& Electronic Engineering, Harbin University of Science and Technology
}

\begin{abstract}
Islanding detection is one of the essential features for photovoltaic grid-connected systems; detection performance is directly related to safe operation of the equipment. Summarize existing islanding detection, then an active method based on frequency - reactive power feedback islanding detection is proposed. Once an islanding condition occurs, the method introducing a load frequency - reactive power characteristics associated component as input of reactive power perturbation, breaks the existing islands of stability point in the case of the power balance. Then it detects the system frequency deviation by real time, and draws into the function of reactive perturbation to form a positive feedback loop. So the frequency drifting is accelerated and the frequency of the output voltage of the photovoltaic grid-connected inverter exceeds a preset threshold value, and then the islanding will be detected. Simulation shows that the proposed method has no detection dead zone and it is fast and has little influence on power quality.
\end{abstract}

Keywords: islanding detection; reactive power; frequency drifting; grid-connected systems; feedback; non-detection zone

\section{Introduction}

Currently, a large combination of grid and distributed generation by many of the world of energy, electricity experts is recognized as able to save investment, reduce energy consumption and improve power system reliability and flexibility of the main ways. It is also the 21 st century power industry development direction proposed in [1]. With the development of smart grid, distributed generation will be heavily used .The access of many distributed generation impact on the entire grid, affecting the safety and stable operation of the grid. After the grid lost power, distributed generation and local load independently operate, which is the so-called island (islanding) phenomenon.

In the case of the local mains supply imbalance, islanding will have more serious consequences. Such as island operation will affect the quality of power supply, even damage power supply equipment ; There is a threat to the island's personal safety of maintenance personnel, as it is generally believed that the main grid is disconnected ,the system will not be charged, and the phase synchronization detection does not work, lead to line tripped again and even damage the system or electrical equipment which connect to the distributed generation with the island system back into the grid .Therefore, the timely islanding operation detection and distributed generation systems isolation from the public point, have a positive impact on safe and stable operation of the power system. The viewpoint is proposed in [2].

Booming of distributed generators industry combined to requirements for islanding detection has led to an intensive research and development of methods for identification of islanding conditions. These methods can be classified in three main categories [3]:

- passive methods

- active methods

- communication based methods 
Communication based methods necessitate the involvement of utility in implementation of islanding detection schemes, making them less favorite for practical implementation. Be-cause communication adds also costs to both DG and the grid infrastructure, these methods are not commonly used today [4$5]$.

Further research on active method of frequency-reactive feedback islanding detection can be in this paper .By studying the inverter and feedback control theory, using abc-dq0 transform frequency reactive power feedback combined with dq0 transform inverter islanding detection method, which indirectly increase mismatch degree between the output of the inverter reactive power and reactive power load, it results in micro-grid frequency variation, so that it can effectively detect islanding, reducing non-detection zone, according to Institute of Electrical and Electronics Engineers (IEEE)Std.1547-2000[6] .

\section{Islanding Detection General Principle}

When the microgrid disconnect from the mains in islanding operation, Distributed Generation (DG) and load power difference will change the frequency, voltage. Switching off the mains, the active or reactive power will be changed, lead to micro-grid frequency and voltage variation, which constitutes a feedback to regulate the inverter active or reactive power and match the load power imbalance increasingly in order to accelerate change of voltage/ frequency, thus quickly detect island. Fig. 1 shows islanding detection principle.

Parallel in the mains, DG is connected to point of common coupling (PCC) of the main power grid, providing power to the load, to get:

$$
\begin{aligned}
& P_{\text {load }}=P+\Delta P \quad Q_{\text {load }}=Q+\Delta Q \\
& P_{\text {load }}=V_{P C C}^{2} / R \\
& Q_{\text {load }}=V_{P C C}^{2}\left(\frac{1}{\omega L}-\omega C\right)
\end{aligned}
$$

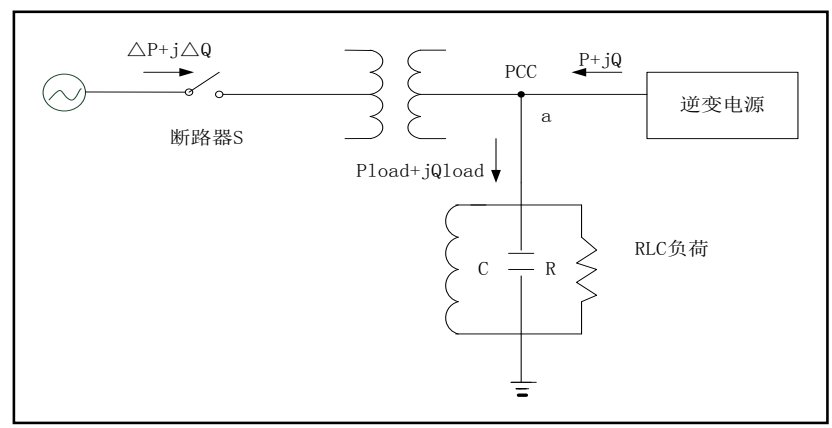

\section{Figure 1. Islanding detection general principle}

Formula: $V_{p c c}$ is common coupling point voltage; $\mathrm{P}, \mathrm{Q}$ is the inverter active, reactive power output; $\Delta P, \Delta Q$ is supplied by the main power grid active power, reactive power. $P_{\text {load }}, Q_{\text {load }}$ is consumed by the load active power, reactive power.

The above three simultaneous equations can be obtained:

$$
\omega=\frac{1}{2 \sqrt{L C}}\left(\sqrt{\left(\frac{Q_{\text {load }}}{Q_{f} P_{\text {load }}}\right)^{2}+4}-\frac{Q_{\text {load }}}{Q_{\mathrm{f}} P_{\text {load }}}\right)
$$


Formula (4), $Q_{f}=R \sqrt{\frac{C}{L}}$ or $Q_{f}=\frac{1}{P} \sqrt{Q_{L} * Q_{C}}$ is the quality factor of load.

On the other hand, the frequency of an islanded feeder can have various values depending on the power mismatch between the load and generation in the island. Excess generation will drive up the frequency and deficit generation will result in the decline of frequency. Accordingly, if there is a large power mismatch in an islanding, the frequency-based antiislanding scheme will be able to detect islanding condition quickly. If the power mismatch is small, however, it will take longer time to detect is landing condition [7].

\section{Frequency feedback in dq axes}

Frequency feedback can be divided into two kinds: Frequency - reactive power feedback and frequency - active power feedback. Switching off the mains before and after, the reactive power of islanding system is changed, while active power is constant, then result in changes of frequency without the voltage variation, it can constitute a frequency - reactive power negative feedback, increasing the mismatch between inverter reactive power output and the load carried, then the frequency can vary continuously until the limit. It is shown in Figure 2.

Analyze and calculate the abc-phase voltage and current into dq components, the following formula:

$$
\left[\begin{array}{l}
\mathrm{i}_{d} \\
i_{q}
\end{array}\right]=C_{d q} C_{32}\left[\begin{array}{l}
i_{a} \\
i_{b} \\
i_{c}
\end{array}\right]=C\left[\begin{array}{l}
i_{a} \\
i_{b} \\
i_{c}
\end{array}\right]
$$

Where $C_{32}$ and $C_{d q}$ are as follows:

$$
\begin{gathered}
C_{32}=\frac{2}{3}\left[\begin{array}{ccc}
1 & -\frac{1}{2} & -\frac{1}{2} \\
0 & \frac{\sqrt{3}}{2} & -\frac{\sqrt{3}}{2}
\end{array}\right] \\
C_{d q}=\left[\begin{array}{cc}
\sin \omega t & -\cos \omega t \\
-\cos \omega t & -\sin \omega t
\end{array}\right]
\end{gathered}
$$

Active power component is proportional to the $\mathrm{d}$ axes, and reactive power is proportional to the q axes. Therefore, the load of active and reactive power demand can feed back to the dq coordinate axes respectively, even voltage and current vectors are the resultant with the respective dq component, so any component vector transformation will cause not only variation in amplitude but also change of the phase difference between dq axes component . As a result, frequency is changed. Applying dq axes transformation to the above feedback. It is shown in Figure 3 and Figure 4. 


$$
\begin{aligned}
& \Delta P=0 \quad Q_{D G}>Q_{\text {load }} \rightarrow Q_{\text {load }} \uparrow \rightarrow \omega \uparrow \rightarrow Q_{c} \uparrow \rightarrow Q_{D G} \uparrow \rightarrow \omega \uparrow \\
& Q_{D G}<Q_{\text {load }} \rightarrow \stackrel{\sqrt{Q_{\text {load }} \downarrow \rightarrow \omega} \rightarrow \rightarrow Q_{c} \downarrow \rightarrow Q_{D G} \downarrow \rightarrow \omega \downarrow}{V^{\prime}}
\end{aligned}
$$

Figure 2. Frequency - reactive power feedback

$$
\begin{aligned}
& P_{D G}>P_{\text {load }} \rightarrow V_{P C C} \uparrow \rightarrow V_{d} \uparrow \rightarrow I_{\text {dref }} \uparrow \rightarrow P_{D G} \uparrow
\end{aligned}
$$

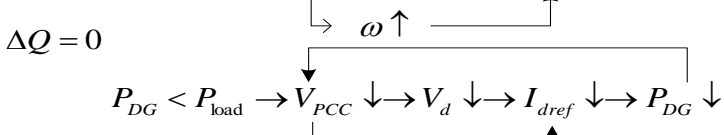

$$
\begin{aligned}
& \omega \uparrow \longrightarrow
\end{aligned}
$$

Figure 3. Dq axes frequency - active power feedback

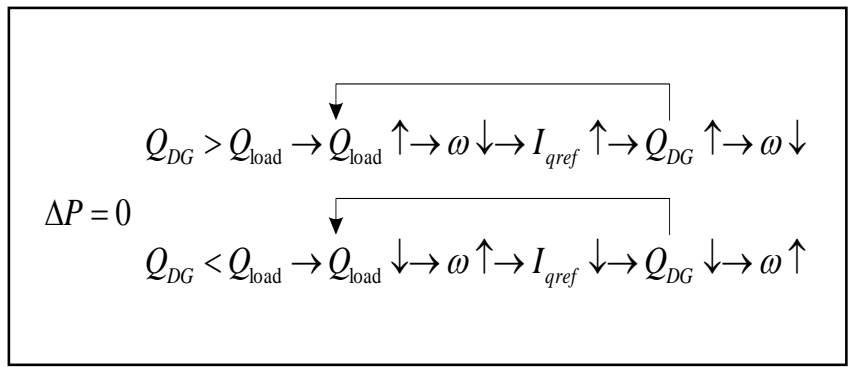

Figure 4. Dq axes frequency - reactive power feedback

For example: Parallel in the mains ,because of the main power grid control, frequency stabilize in the power frequency with no changes at this time; Switching off the mains, the power difference will cause change of the frequency micro-grid, if the inverter perceive the decrease of PCC frequency, it will regulate the q axes current reference component increase accordingly, resulting in a corresponding addition in inverter reactive power output. As a result, frequency continues to decline, forming a feedback, and frequency varies constantly in one direction until the limited frequency finally, thus it can detect the islanding. Even a slight difference can be detected by the introduction of feedback in this case. Referring to Figure 5, when $V_{d}$ or $V_{q}$ occurs disturbances, it will affect the voltage vector amplitude and angle, and frequency is changed close along with angle variation. If there is a single component as the inverter power factor is 1 , the angle is constant.

Analysis shows that application of feedback in the dq axes, can produce a range of feedback programs: 


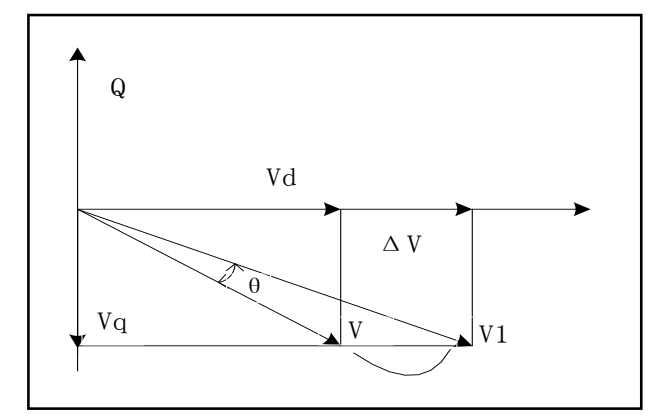

Figure 5. Dq axes component of $\mathrm{V}$

for example $V_{d}$ to $I_{\text {dref }}, V_{d}$ to $I_{\text {qref }}, V_{q}$ to $I_{\text {dref }}, V_{q}$ to $I_{\text {qref }}, \omega$ to $I_{\text {dref }}, \omega$ to $I_{\text {qref }}$. In connection with feedback and application of concepts, this paper designs a $\omega$ to $I_{\text {qref }}$ feedback. $I_{d r e f}$ is Id benchmark reference volume in q axes. The following formula:

$$
I_{\mathrm{qref}}(i)=K\left(\omega_{0}-\omega_{u}(i)\right)+i_{q r e f}
$$

Of which: $i_{q r e f}$ is q-axis reference islanding current .If the inverter run with unity power factor $i_{\text {qref }}$ is $0 ; \omega_{0}$ is the power frequency, $\omega_{u}$ is the frequency of the PCC voltage , K is the gain.

Parallel in the mains, the micro-grid supported by a large grid, generally stabilize in frequency $50 \mathrm{~Hz}$, current reference phase in q coordinate axes $I_{\text {qref }}=i_{\text {qref }}$, feedback does not affect circuitry at this time. Once switching off the mains, power inverter and carried load have frequency / voltage variation because of a slight difference in power, and q axes current reference phase is changed by a slight difference in frequency changes since the presence of frequency - reactive power feedback, so that both DG reactive power output and frequency are changed. Frequency varies constantly in one direction until the limited frequency finally, thus it can detect the islanding [8]. See Figure 6:

$$
\begin{aligned}
& Q_{D G}>Q_{\text {load }} \rightarrow \varpi \downarrow \rightarrow I_{\text {qref }} \uparrow \rightarrow Q_{D G} \uparrow \rightarrow \omega \downarrow \\
& \Delta P=0 \quad \begin{array}{l}
\downarrow \\
Q_{D G}<Q_{\text {load }} \rightarrow \omega \uparrow \rightarrow I_{\text {qref }} \downarrow \rightarrow Q_{D G} \downarrow \rightarrow \omega \uparrow
\end{array}
\end{aligned}
$$

\section{Figure 6. Dq axes frequency reactive power feedback}

\section{Frequency - reactive power feedback islanding detection simulation}

Parallel in the mains, detected frequency remains unchanged since frequency of the main power grid is constant. Switching off the mains, once the inverter output and the load reactive power requirements do not match, frequency of the load will vary. The experimental simulation model is designed by matlab/simulink. As shown in Figure 7.

Propose the load reactive power requirements in this paper: Inverter system providing only part of reactive power, the rest is still provided by the main power grid. It ensures that the 
inverter reactive power output and load requirements are inconsistent when islanding appears, leading to change of the frequency in one direction until the limited frequency finally. System simulation experiment time is set to $1.0 \mathrm{~s}$. And at $0.2 \mathrm{~s}$, switching off the mains, i.e., it is islanding state. The theory is proposed in [9-10].

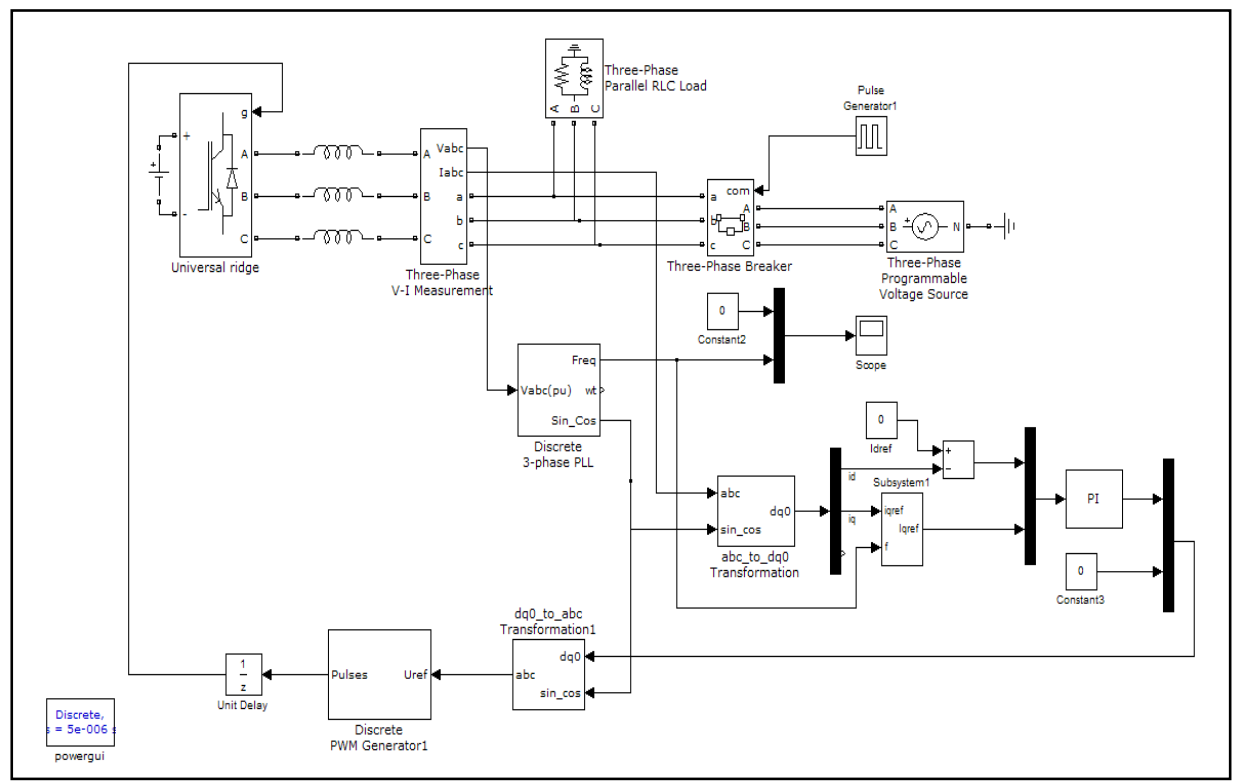

Figure 7. Frequency - reactive feedback islanding detection simulation diagram

\section{Simulation Results Analysis}

Parallel in the mains, the voltage and frequency at point of common coupling maintained constant which are constrained by main power grid. Distributed Generation only need to provide active or reactive power during parallel mode.

Simulation results, as shown in Figure 8. The measured three-phase current through the abc-dq0 converter, convert to $I_{d}, I_{q}$. The two differences are obtained between $I_{q}$ and the inverter feedback q axes current reference quantity $I_{\text {qref }}$ and $I_{\text {dref }}$, through a proportionalintegral (PI) controller, with dq0-abc converter, thus turn into the reference sinusoidal pulse width modulated wave (SPWM), thereby control DG inverter output. In addition, PI regulator can reduce the instability caused by feedback for system, and phase locked loop on the one hand can supply load current and converter output current by SPWM modulation wave with consistent reference phase, on the other hand can record voltage and frequency even obtain frequency variation.

$I_{d r e f}$ : Set the DG output power $\mathrm{P}$, then by $P=3 U I \cos \phi,(\cos \phi$ is the power factor $)$, to obtain the current value $I_{d r e f}$, then with abc-dq0 conversion, calculated $I_{d r e f}$. Design $\cos \varphi=$ 0.5 .

$I_{\text {qref }}$ : As is shown in Figure 9, the measured frequency got through low-pass filter, differenced with power frequency, and then multiplied by the gain factor of the feedback, through the limiter, to obtain $I_{\text {qref }}$. Wherein: the low-pass filter is used to eliminate the effect of noise (high-frequency signals). 


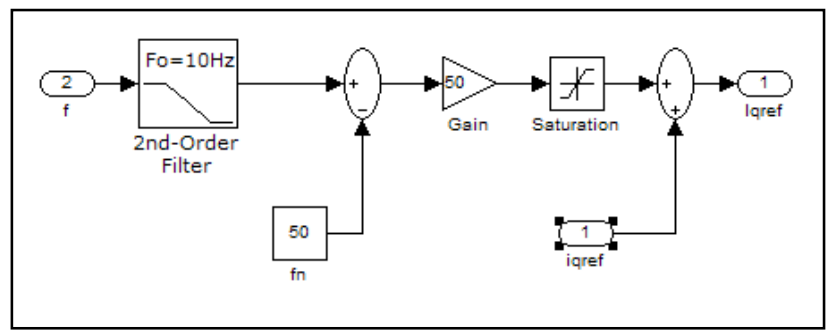

Figure 8. Simulink simulation diagram

Selection of the feedback loop gain should be considered two cases: Parallel in the mains, gain should be small enough so that the system can run in a stable state, then set $\mathrm{k}=1$. Switching off the mains, gain requires to be large enough to make the island the system unstable, so as to cause voltage and frequency variation, finally detect islanding state, set $\mathrm{k}=$ 50. Otherwise, the frequency/voltage are no more limited, the islanding will enter another stable state, so it cannot be detected, also generate non-detection zone. Limiter is used to control feedback maximum gain, which is considered the inverter maximum current limit in the design process.

In this experiment load active power is set to $\mathrm{P}=10 \mathrm{~kW}$, by $P=3 U I \cos \phi, \cos \phi=0.5$, calculated $I_{\text {dref }}=47.2$. Theoretically the value is suited, the best data is obtained by trial and $I_{d r e f}=49.2$.

(1) Load reactive power is set to $\mathrm{Q}=900 \mathrm{~W}$, at this time $Q_{D G}>Q_{\text {load }}$, there is a theoretical analysis $Q_{D G}>Q_{\text {load }} \rightarrow \omega \downarrow \rightarrow I_{\text {qref }} \uparrow \rightarrow Q_{D G} \uparrow \rightarrow \omega \downarrow$, until frequency exceeds the limit, so that it detected islanding. From Figure 9 can be found that parallel in the mains frequency stabilizes in $50 \mathrm{~Hz}$ or so, $0.2 \mathrm{~s}$ switching off the mains and then put into the feedback, accelerate change of frequency, the frequency decreases gradually, exceeding the limit when at $0.32 \mathrm{~s}$, it can effectively detect the islanding condition. According to IEEE std.929-2000 [11], combined with the domestic situation, the lower limit of the normal operating frequency of $49.3 \mathrm{~Hz}$.

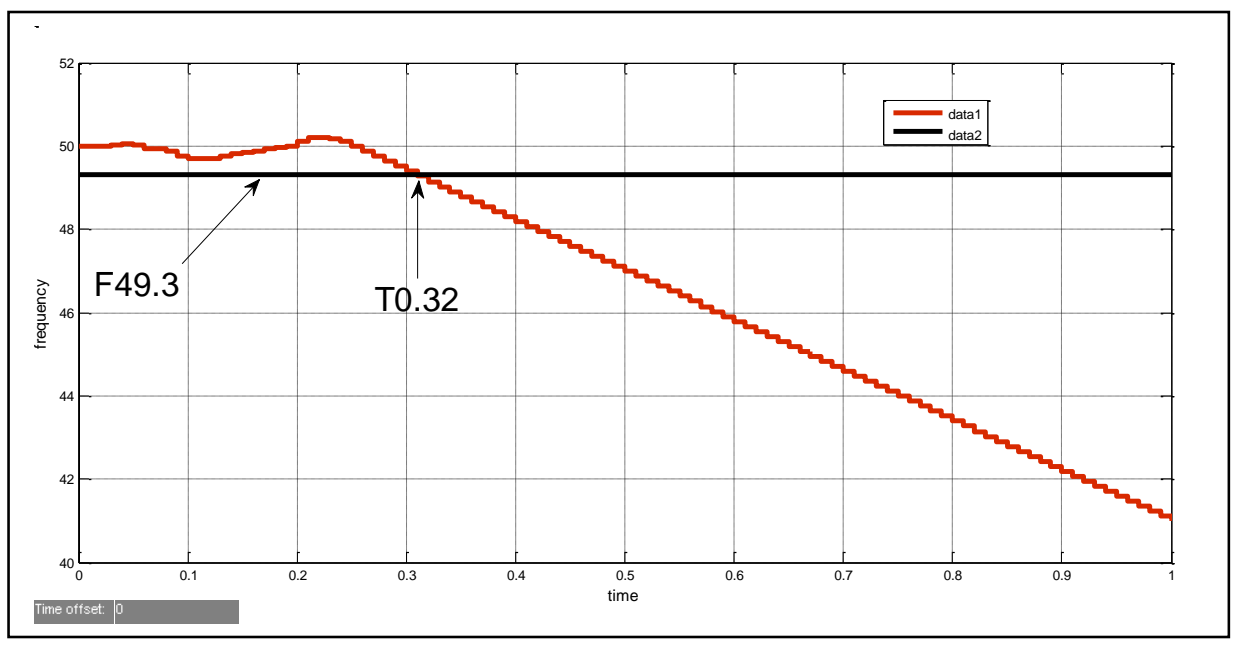

Figure 9. Reactive power setting value, the inverter frequency curve 
(2) Load reactive power is set to $\mathrm{Q}=1000 \mathrm{~W}$, at this time $Q_{D G}<Q_{\text {load }}$, there is a theoretical analysis $Q_{D G}>Q_{\text {bad }} \rightarrow \omega \downarrow \rightarrow I_{\text {qref }} \uparrow \rightarrow Q_{D G} \uparrow \rightarrow \omega \downarrow$, until frequency exceeds the limit, so that it detected islanding. From Figure 10 can be found that parallel in the mains frequency stabilizes in $50 \mathrm{~Hz}$ or so, $0.2 \mathrm{~s}$ switching off the mains and then put into the feedback, accelerate change of frequency, the frequency increases gradually, exceeding the limit when at $0.23 \mathrm{~s}$, it can effectively detect the islanding condition. According to IEEE std.929-2000, combined with the domestic situation, the lower limit of the normal operating frequency of $50.5 \mathrm{~Hz}$.

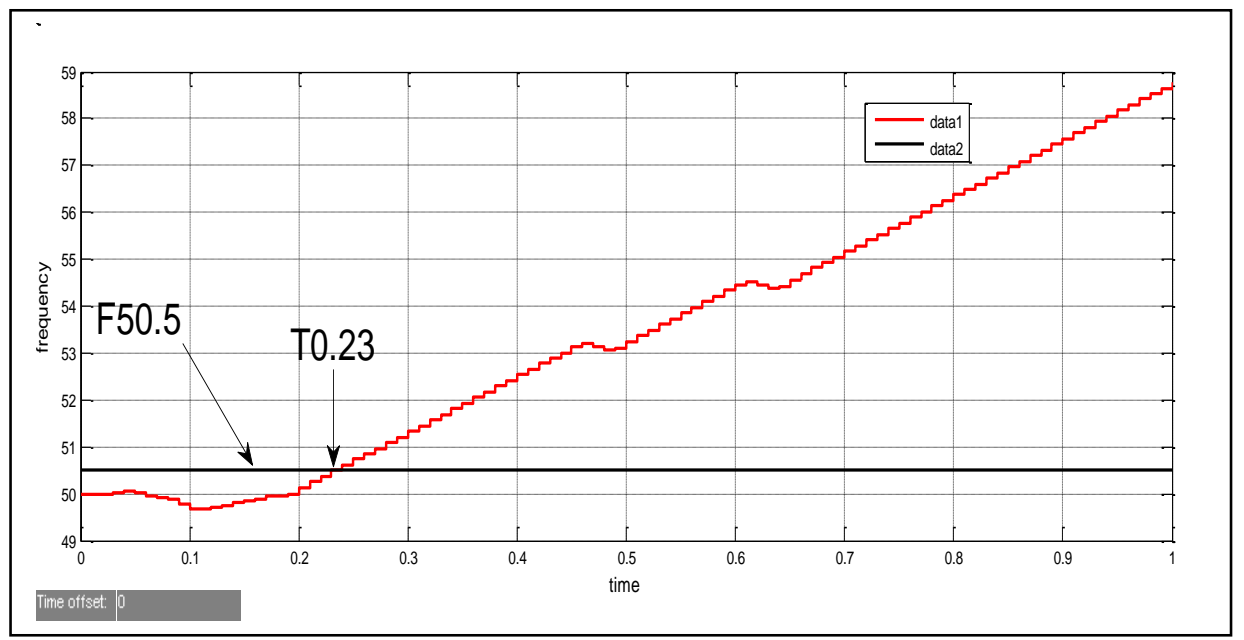

Figure 11. Reactive power setting value, the inverter frequency curve

\section{Conclusion}

Elaborate design of frequency - reactive power feedback inverter islanding detection method based on principle of $\mathrm{dq}$ axes, firstly introduce the principle of the frequency reactive power feedback based on $\mathrm{dq}$ axes transformation, secondly design the feedback frequency conversion to $I_{\text {qref }}$ and finally MATLAB / SIMULINK simulation prove the effectiveness of this method: When DG inverter output power and load power are close, it can effectively reduce non-detection zone, actually indirectly increase mismatch degree between inverter reactive power output and reactive power load, so that frequency varies constantly in one direction until the limited frequency finally, so as to detect the islanding. The method utilizes feedback theory, increases forcibly mismatch degree between inverter reactive output and load reactive power in islanding state, keep frequency changing in one direction, until the limit. This method is simple, easy to implement, and is suitable for Multi-Distributed Generation.

\section{Acknowledgements}

The work was supported by Nature Science Foundation of Heilongjiang Province, China (E200932).The results are also part of innovative experimental subjects and initial achievement research in islanding detection method. 


\section{References}

[1] D. Ming and W. Min, "Distributed generation technologies", Electric Power Automation Equipment, vol. 24, no. 7, (2004), pp. 31-35.

[2] Y. Yu, L. Xinchao, F. Chen and W. Peng, "Smart Grid Development islanding detection control unit", State Grid Electric Power Research Institute, Electric Power Construction, vol. 30, no. 9, (2009), pp. 5-7.

[3] H. H. Zeineldin, J. L. Kirtley, "Islanding operation of inverter based distributed generation with static load models", 2008 IEEE Power and Energy Society General Meeting-Conversion and Delivery of Electrical Energy in the 21st Century, (2008), Pittsburgh, PA, USA.

[4] M. A. Pefern, O. Usta and G. Fielding, "Protection against loss of utility grid supply for a dispersed storage and generation unit", IEEE Transactions on Power Delivery, vol. 8, no. 3, (1993).

[5] C. J. Mozina, "Interconnection protection of IPP generators at commercial/industrial facilities", IEEE Transactions on Industry Applications, vol. 37, no. 3, (2001).

[6] IEEE Std.1547-2003, "IEEE Standard for interconnecting Distributed resources with electric", (2003).

[7] W. Xu, K. Mauch and S. Martel, "An Assessment of Distributed Generation Islanding Detection Methods and Issues for Canada”, CANMET Energy Technology Centre-Varennes, Natural Resources Canada, (2004) July.

[8] N. Chongxuan, "Islanding detection and island divided", Tianjin University/Power System and Automation, (2008), pp. 31-34.

[9] O. Samuelsson and N. Strath, "Islanding detection and connection requirements", Power Engineering Society General Meeting, IEEE, (2007) June 24-28, Tampa, Florida.

[10] S. Meiling, "Based on the positive feedback islanding detection of inverter", Yanshan University/Circuits and Systems, (2007), pp. 48-52.

[11] IEEE Std.929-2000, "IEEE Recommended Practice for Utility Interface of Photovoltaic(PV) Systems", Institute of Electrical and Electronics Engineers, Inc., New York, USA, (2000). 
International Journal of Grid and Distributed Computing Vol. 6, No. 4, August, 2013 